\title{
Changes in the H-Reflex Amplitude Caused by Fluctuation of Stimulus Frequency
}

\author{
Shigeyuki ImURA ${ }^{1)}$, Michiaki Kishikawa ${ }^{2)}$, Tomohiro WadA ${ }^{1)}$, Akihiko Iwai ${ }^{3)}$, \\ MaKoto FuZiwara, MD ${ }^{1)}$ \\ ${ }^{1)}$ Rehabilitation Center, Hyogo College of Medicine Hospital: 1-1 Mukogawa-cho, \\ Nishinomiya, Hyogo 663, Japan. TEL +81 798-45-6358. \\ ${ }^{2)}$ Chubu Rehabilitation College \\ ${ }^{3)}$ Watanabe Orthopaedic Clinic
}

\begin{abstract}
We investigated the changes in the H-reflex amplitude at various stimulus frequencies, in order to identify the stable index in a relatively short period. 10 subjects volunteered for experiment. The H-reflex was obtained from the left soleus. The $\mathrm{H}$ wave was induced by 29 degrees of stimulus frequency: 10 degrees from 0.1 to $1.0 \mathrm{~Hz}$ with an increase of $0.1 \mathrm{~Hz}$, and 19 degrees from 1.0 to $20.0 \mathrm{~Hz}$ with an increase of $1.0 \mathrm{~Hz}$. The decrease in $\mathrm{H}$ wave amplitude tended to become greater with an increase in stimulus frequency. The frequency depression curve at stimulus frequencies from 1.0 to $20.0 \mathrm{~Hz}$ indicated a significant frequency depression $(p<0.01)$ with an increase in stimulus frequency. Disinhibition of the H-reflex amplitude at stimulus frequencies from 4.0 to $8.0 \mathrm{~Hz}$ is a specific response, and the change rate of the $\mathrm{H}$-reflex amplitude at the stimulus frequency of $6.0 \mathrm{~Hz}(62.9 \%)$ was almost the same as that of $1.0 \mathrm{~Hz}(62.5 \%)$. We found that repetitive evoked EMG using a stimulus frequency of approximately $5 \mathrm{~Hz}$ is a great value in examining the change in the H-reflex amplitude and has a good possibility of clinical application.
\end{abstract}

Key words: H-reflex, repetitive evoked EMG, frequency depression curve

(This article was submitted Jul. 12, 1997, and was accepted Nov. 10, 1997)

The Hoffman reflex (H-reflex) is generally accepted as a spinal monosynaptic reflex, and also as a muscle proprioceptive reflex observed in evoked electromyograms (EMG). Therefore the H-reflex is widely used as one of the indices for spinal motoneuron excitability. In recent years, there has been a report describing that the H-reflex mirrors the activities of multiple motoneurons, not single ones, and fluctuates according to voluntary movements ${ }^{1)}$. This suggests that the H-reflex possesses not only the character of a spinal motoneuron, but also the physiological character of a spinal motoneuron pool.

The H-reflex can be obtained by a simple procedure, and measured from a conscious subject with no motor stress. Examination of $\mathrm{H}$-reflex amplitude changes allows us to clarify the effects of various exercise therapy maneuvers such as the PNF technique ${ }^{2-4)}$, tendon pressure ${ }^{5) 6)}$, muscle stretching7)8), manual muscle massage ${ }^{9-11)}$, and heat/cold therapy ${ }^{12) 13)}$, on the human spinal motoneuron pool. The H-reflex is usually examined at a certain stimulus frequency, and the average amplitude is used to obtain stable results. However the effects of each stimulus remain for several seconds, and the question then arises about the frequency of repetitive stimuli. A long stimulus interval can reduce the effect of a previous stimulus, but requires a longer experiment time, which may result in fatigue and a change in the mental condition of the subjects. The stimulus frequency should therefore be decided according to the purpose of the examination. The stimulus frequency of $0.1 \mathrm{~Hz}$ is recommended in the H-reflex Examination Guideline (1973)14), but the 
interval is too long to be used in a clinical context. Stimulus frequencies from 0.2 to $0.5 \mathrm{~Hz}$ have recently been applied.

The amplitude of the H-reflex, induced by repetitive stimuli at a certain interval, has been found to decrease gradually until the third stimulus and reaches a plateau thereafter. Although a large number of studies have been made on the effect of repetitive stimuli on the monosynaptic reflex in animal spinal cords ${ }^{15-18)}$, the recovery curve by double stimuli, the repetitive evoked electromyogram, and frequency depression curve, are mainly applied to human studies, and no systematic investigation has ever tried to characterize the change in amplitude. Therefore, our study was designed to investigate the changes in the H-reflex amplitude at various stimulus frequencies, and to identify the stable index of the H-reflex in a relatively short period.

\section{METHODS}

Table 1 demonstrates the physical characteristics and occupations of 10 subjects ( 6 males, 4 females; average age: $25.3 \pm 6.7$ years). Prior to participation, a full explanation was given to all subjects, and their consent was obtained.

The subjects positioned themselves in a supine position to avoid change in muscle length and tone. They were instructed to relax their triceps surae to the appropriate extent, with the knee joint flexed at 30 degrees and the ankle free. During measure-

Table 1. Characteristics of subjects and occupation.

\begin{tabular}{ccccc}
\hline Subject & $\begin{array}{c}\text { Age } \\
\text { (years) }\end{array}$ & Sex & $\begin{array}{c}\text { Dominant } \\
\text { Hand }\end{array}$ & Occupation \\
\hline (1) & 20 & $\mathrm{M}$ & $\mathrm{Rt}$ & $\mathrm{S}$ \\
$(2)$ & 26 & $\mathrm{M}$ & $\mathrm{Rt}$ & $\mathrm{PT}$ \\
(3) & 26 & $\mathrm{~F}$ & $\mathrm{Rt}$ & $\mathrm{PT}$ \\
(4) & 24 & $\mathrm{M}$ & $\mathrm{Rt}$ & $\mathrm{PT}$ \\
(5) & 21 & $\mathrm{~F}$ & $\mathrm{Rt}$ & $\mathrm{S}$ \\
(6) & 43 & $\mathrm{M}$ & $\mathrm{Rt}$ & $\mathrm{PT}$ \\
(7) & 21 & $\mathrm{~F}$ & $\mathrm{Rt}$ & $\mathrm{S}$ \\
(8) & 25 & $\mathrm{M}$ & $\mathrm{Rt}$ & $\mathrm{PT}$ \\
(9) & 21 & $\mathrm{M}$ & $\mathrm{Rt}$ & $\mathrm{S}$ \\
(10) & 26 & $\mathrm{~F}$ & $\mathrm{Rt}$ & $\mathrm{OT}$ \\
\hline & 25.3 & $\mathrm{M} 6$ & $\mathrm{Rt} 10$ & \\
& \pm 6.7 & $\mathrm{~F} 4$ & & \\
\hline
\end{tabular}

M: Male, F: Female, Rt: Right, PT: Physical Therapist, OT: Occupational Therapist, S: Student ments, subjects were asked not to move and to relax their mental state by reading figures from 1 to 100 silently. External stimuli such as sudden noise or light were minimized as much as possible.

The experiment was conducted in a temperature controlled $\left(27.0 \pm 0.8^{\circ} \mathrm{C}\right)$ room for electromyographic examination. The H-reflex was induced by a pair of surface electrodes (SEE 103 Dish electrode, NEC Sanei) placed vertically $3 \mathrm{~cm}$ apart along the belly of the left soleus. Prior to placing the electrodes, the skin surface was cleaned with a sand paste to remove dirt and keratin, in order to reduce skin resistance below $10 \mathrm{k} \Omega$. Using a stimulation electrode, the posterior tibial nerve was percutaneously stimulated at the popliteal crease, and the point which indicated the lowest H-reflex threshold was identified. The stimulation electrodes were then fixed tightly by a felt band with a pad but not so as to affect peripheral circulation. Indifferent electrodes were fixed with surgical tape at the proximal site of the suprapatella edge, so that the electric current could be applied vertically to the nerve.

Constant electric stimulation was used to obtain a stable stimulatory effect, and a 1-msec square wave was used to make a sufficient difference from the threshold for the $\mathrm{M}$ wave, and to minimize the artifact.

The threshold value of the H-reflex of double stimuli with a 6-msec interval was obtained, and 1.12-fold of the threshold value was used as the standard for stimulus intensity. This stimulus intensity is a little greater than that required to elicit the H-reflex by a single stimulation, but not strong enough to evoke a minimal M-wave. Constancy of stimulus intensity was certified by re-examining the threshold of the $\mathrm{H}$ wave with a monitor.

The $\mathrm{H}$ wave was induced by 29 degrees of stimulus frequency; 10 degrees from 0.1 to $1.0 \mathrm{~Hz}$ with an increase of $0.1 \mathrm{~Hz}$, and 19 degrees from 1.0 to $20.0 \mathrm{~Hz}$ with an increase of $1.0 \mathrm{~Hz}$. Ten consecutive $\mathrm{H}$ waves were recorded at each stimulus frequency. The order of frequency was randomized to avoid projection of each stimulus, and the average amplitude for 3 measurements was adopted.

An electromyograph (MYSTRO MS-25 type, Medelec Co.) was used to induced the H-reflex and to employ the stimulus. The data were collected by a data recorder (TEAC R-60) and analyzed by the bio-information multitask analyzing program 
(BIMUTAS, Kissei Comtec.). ANOVA was used for analysis of difference in each experiment.

\section{RESULTS}

The change rates of the H-reflex amplitude by the repetitive evoked EMG at stimulus frequencies from 0.1 to $1.0 \mathrm{~Hz}$, and 1.0 to $10.0 \mathrm{~Hz}$, and 11.0 to $20.0 \mathrm{~Hz}$ are shown in Fig. 1, 2, 3, respectively.
The H-reflex amplitudes of 10 consecutive waves are expressed as a percentage of the first amplitude. With increasing stimulus frequency, the decrease in the H-reflex amplitude became greater after the 2 nd wave. A significant $(\mathrm{p}<0.01)$ decrease in the amplitude between the 1 st and 2 nd waves was observed at all the stimulus frequencies, except $0.1 \mathrm{~Hz}$. The change rate of the $\mathrm{H}$-reflex at stimulus frequencies from 0.1 to $8.0 \mathrm{~Hz}$
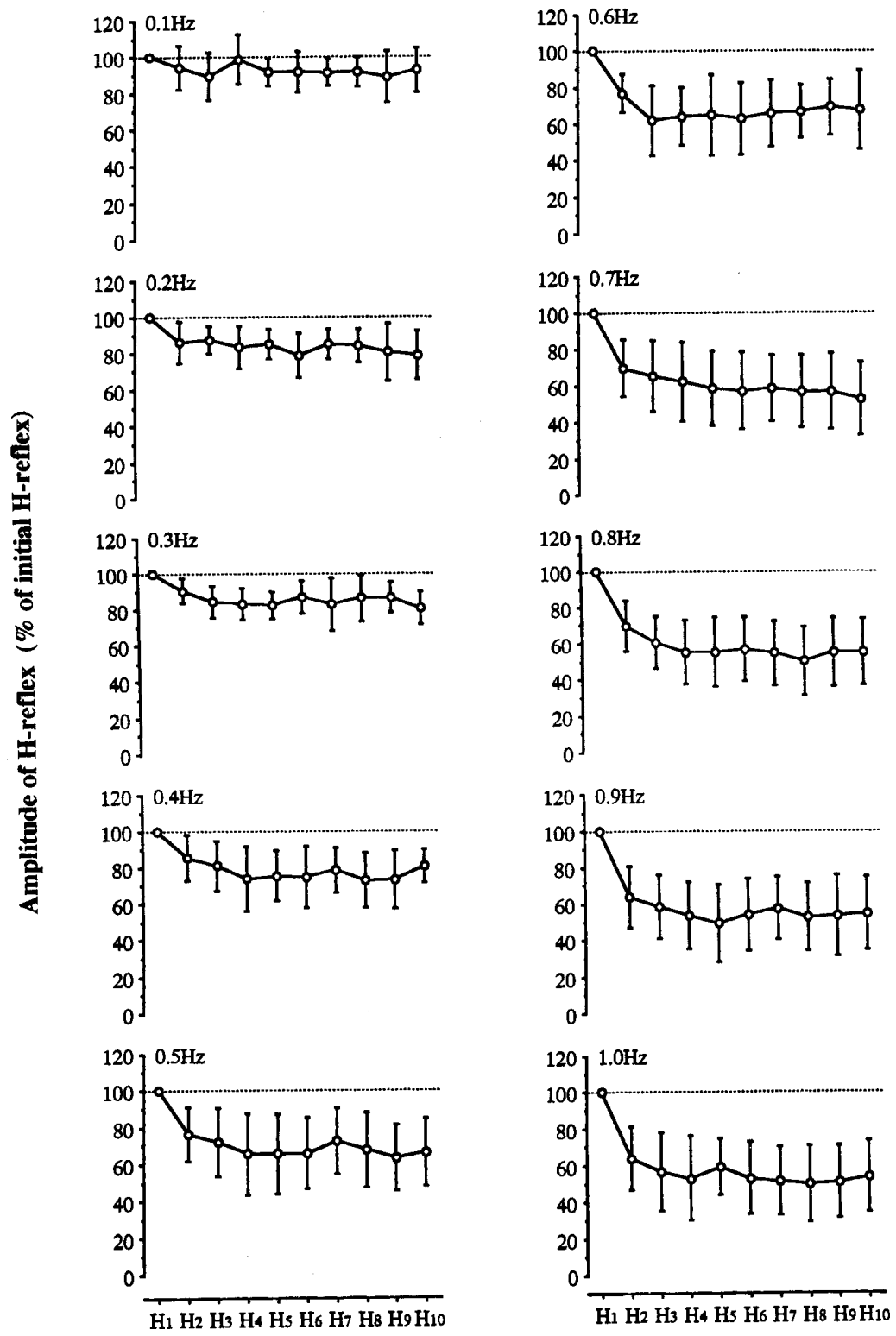

Fig. 1. Change rate of the H-reflex amplitude by the repetitive evoked EMG at stimulus frequencies from 0.1 to $1.0 \mathrm{~Hz}$. A significant decrease $(\mathrm{p}<0.01)$ in the amplitude was observed at all stimulus frequencies except $0.1 \mathrm{~Hz}$. Values are mean \pm SD. 

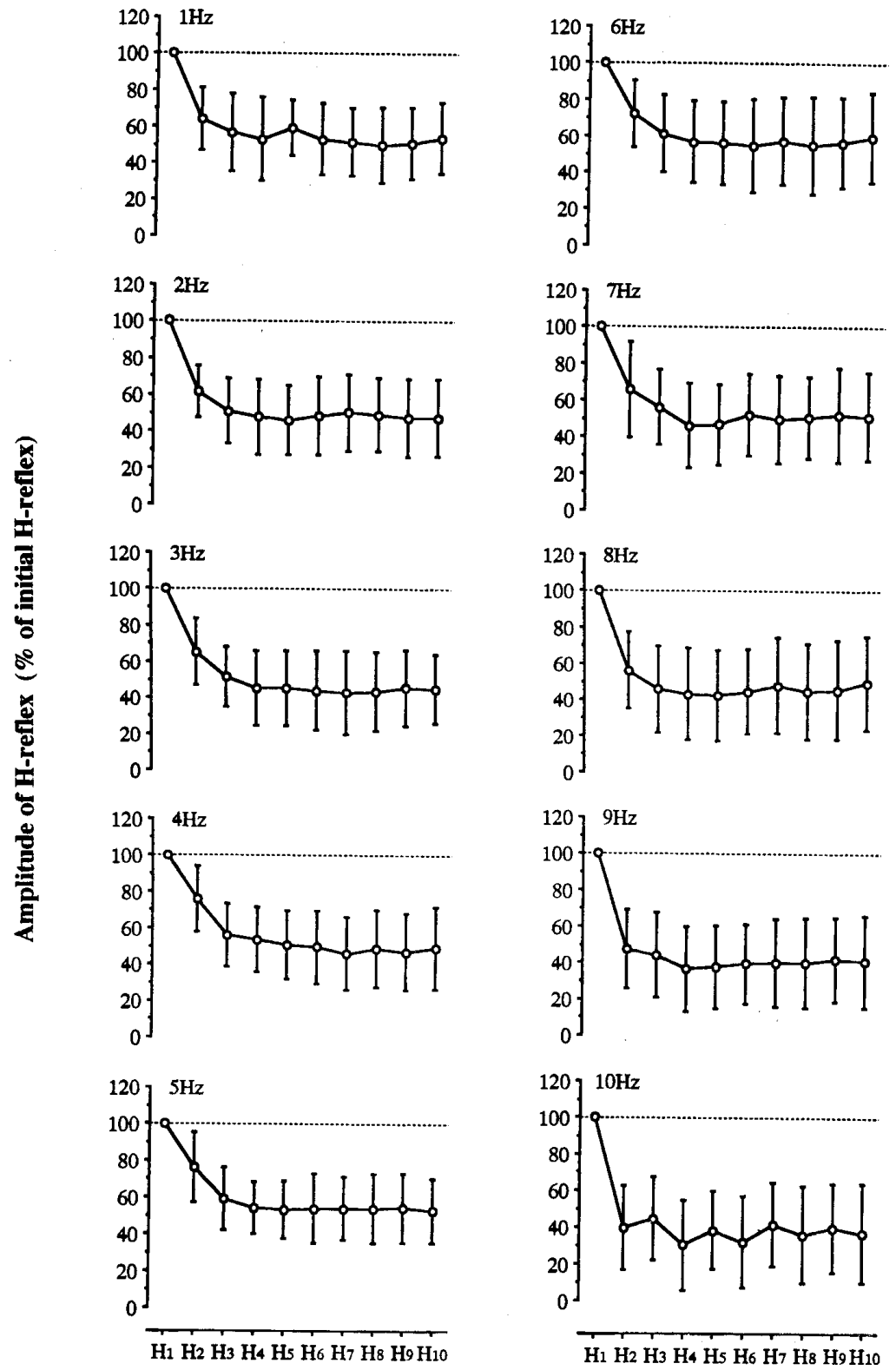

Fig. 2. Change rate of the H-reflex amplitude by the repetitive evoked EMG at stimulus frequencies from 1.0 to $10.0 \mathrm{~Hz}$. A significant decrease $(\mathrm{p}<0.01)$ in the amplitude was observed at all stimulus frequencies. Values are mean \pm SD.

demonstrated a descending curve with a gentle slope until the 4th wave, and almost levelled off from 5 th wave. At stimulus frequencies from 9.0 to $10.0 \mathrm{~Hz}$, a marked inhibition of the H-reflex amplitude was indicated at the 2 nd wave. Inhibition and disinhibition of the H-reflex amplitude appeared reciprocally at the stimulus frequencies from 10.0 to $15.0 \mathrm{~Hz}$. The decrease in the amplitude tended to become greater with an increase in stimulus frequency, but the change rates of the $\mathrm{H}$ reflex amplitude of the 2 nd wave at the stimulus frequencies of $4.0 \mathrm{~Hz}(76.2 \%)$ and $5.0 \mathrm{~Hz}(76.2 \%)$ were greater than those of $1.0 \mathrm{~Hz}(67.9 \%), 2.0 \mathrm{~Hz}$ $(61.6 \%)$ and $3.0 \mathrm{~Hz}(65.3 \%)$.

Figure 4 depicts the method for obtaining the representative value of frequency depression at each stimulus frequency. The frequency depression curve was drawn using the ratio of the average 


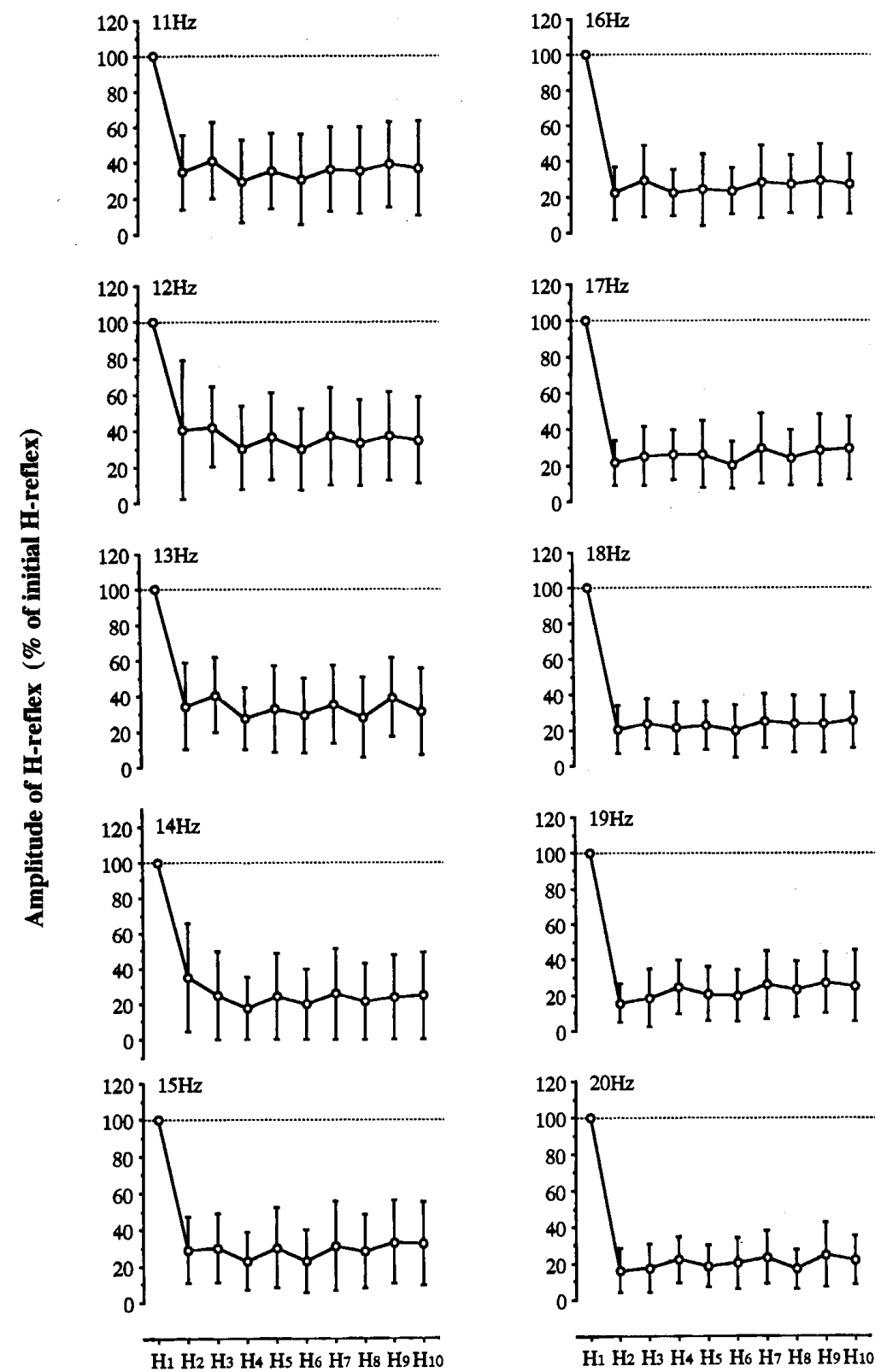

Fig. 3. Change rate of the H-reflex amplitude by the repetitive evoked EMG at stimulus frequencies from 11.0 to $20.0 \mathrm{~Hz}$. A significant decrease $(\mathrm{p}<0.01)$ in the amplitude was observed at all stimulus frequencies. Values are mean \pm SD.

value of the 2 nd to 10 th amplitudes to the 1 st amplitude (Fig. 5, 6). As observed in Fig. 5, a significant frequency depression $(\mathrm{p}<0.01)$ was noted at stimulus frequencies from 0.1 to $1.0 \mathrm{~Hz}$ with increasing stimulus frequency. The frequency depression curve at stimulus frequencies from 1.0 to $20.0 \mathrm{~Hz}$ indicated a significant frequency depression $(\mathrm{p}<0.01)$ with an increase in stimulus frequency (Fig. 6). Disinhibition of the H-reflex amplitude at stimulus frequencies from 4.0 to 8.0 $\mathrm{Hz}$ is a specific response, and the change rate of the H-reflex amplitude at the stimulus frequency of $6.0 \mathrm{~Hz}(62.9 \%)$ was almost the same as that of 1.0 $\mathrm{Hz}(62.5 \%)$. 
〈No. of times〉

$\langle$ H-reflex $\rangle$

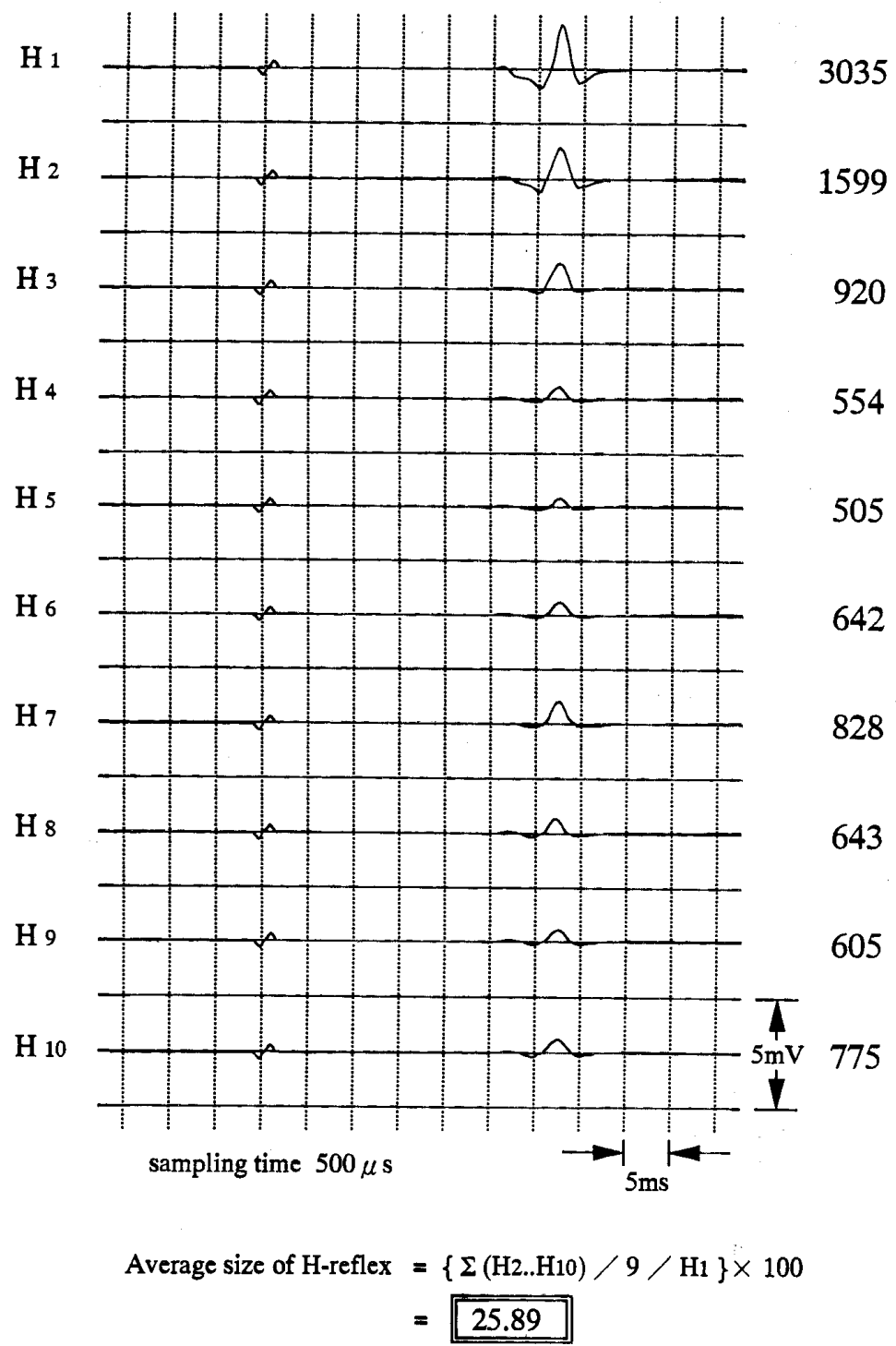

Fig. 4. Method of calculation for the representative value of frequency depression at each stimulus frequency.

\section{DISCUSSION}

The study of the H-reflex pioneered by Hoffmann ${ }^{19)}$ has been extended to various clinical examinations ${ }^{20) 21)}$, with the initial study using the evoked EMG by Magladery. Magladery speculated that the recovery curve of the H-reflex was highly dependent on the change in spinal motoneuron excitability caused by cell combustion, which mainly consisted of an absolute refractory period, relative refractory period, and Renshaw depression. Ookuma et al. reported that during the overresponse period at pulse frequencies from 200 to $300 \mathrm{msec}$, the change in the H-reflex was influenced by the subject's position with the ankle fixed ${ }^{22}$. Morita et al. point out that these changes are provoked not only by changes in spinal cells, but also by the effect of muscle contraction due to the initial stimulus, and changes in nerve fiber excitability of the stimulated region and in neuromuscular trans$\mathrm{fer}^{23)}$. It is therefore thought that a re-examination 


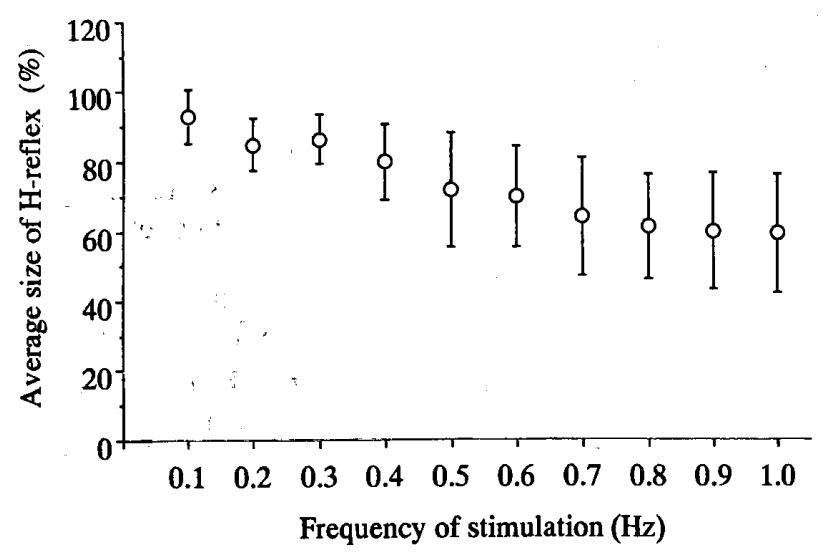

Fig. 5. Frequency depression curve at stimulus frequencies from 0.1 to $1 \mathrm{~Hz}$. A significant frequency depression $(\mathrm{p}<0.01)$ was shown with increasing stimulus frequency. Values are mean \pm SD.

of the application of the recovery curve of the Hreflex to the examination of spasticity is necessary. Honma et al. also point out the difference in the Hreflex response among the stimulus frequencies from 1 to $5 \mathrm{~Hz}^{24)}$.

Hori et al. and Ioku et al. employed consecutive stimuli to the tibial nerve at various stimulus frequencies, and found out that the H-reflex amplitude demonstrated the characteristic pattern for each abnormality of muscle tonus, which stemmed from various neurological disorders. A number of previous researchers have reported that the data of repetitive evoked EMG and frequency depression curve, accord with the clinical findings of patients with neurological disorders ${ }^{25-37)}$, and the data is therefore considered more reliable compared with the results of the recovery curve of the H-reflex, elicited by double stimuli.

When H-reflex induced by repetitive stimuli at various frequencies, the H-reflex amplitude is reported to be decreased in the 2nd and 3rd stimuli, and to level off after several stimuli27)38)39). The initial decrease becomes sharper with increasing stimulus frequency.

This may be attributed to the aftereffects of previous stimulus on the next response, and the present study has shown similar results (Fig. 2, 3). We therefore speculate that the lower the stimulus frequency applied, the smaller the aftereffect of previous stimulus. As indicated in Fig. 1, the aftereffect became too small to be identified at the stimulus frequency of $0.2 \mathrm{~Hz}$ and disappeared at

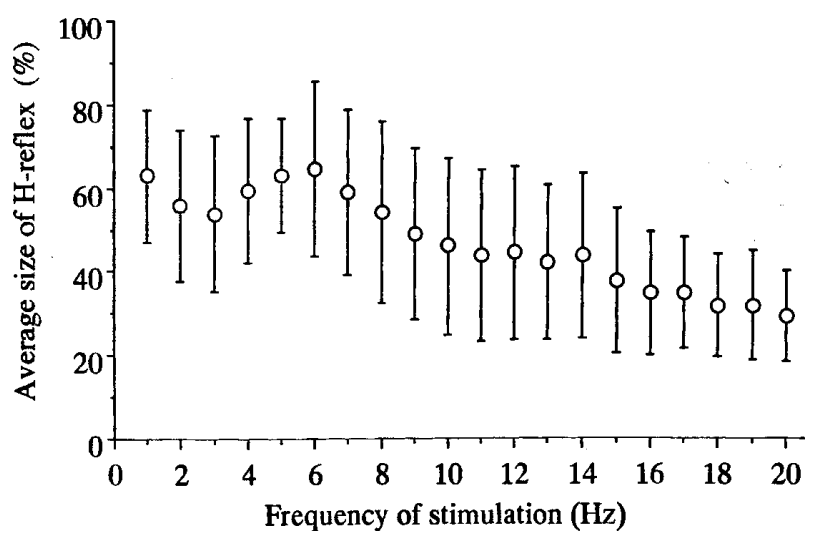

Fig. 6. Frequency depression curve at stimulus frequencies from 1 to $20 \mathrm{~Hz}$. A significant frequency depression $(\mathrm{p}<0.01)$ was shown with increasing stimulus frequency. Values are mean $\pm \mathrm{SD}$.

$0.1 \mathrm{~Hz}$. The primary reason why the H-reflex amplitude is affected by the stimulus frequency is a depletion/decrease of chemical transfer material at the terminal part of Ia group fiber. This may account for a decrease in amplitude of excitatory postsynaptic potential (EPSP) induced by repetitive stimuli. Presynaptic inhibition due to the stimulation of Ia group fiber, and reflex inhibition of the long latent period are possibly responsible for the change in the H-reflex amplitude. The results of our study support this possibility (Fig. 3): inhibition and disinhibition appeared reciprocally from the 2nd wave at the high stimulus frequencies over $10.0 \mathrm{~Hz}$. This response, together with a change in postsynaptic membrane potential, may be the key to the change in the H-reflex amplitude. In animal experiments, the H-reflex is examined under anesthesia or with the body fixed by cordotomy, whereas in humans, it is investigated in an arousal condition by EMG. The effects of muscle contraction caused by the previous stimulus or of mechanical movement are unavoidable in human examination.

Although the effects of previous stimulus are minimized at stimulus frequencies below $0.1 \mathrm{~Hz}$, it has been reported that extremely low frequencies make not only the concentration of the subject lower but also the effect from the outside environment greater, thereby potentially increasing the change in the H-reflex amplitude ${ }^{40) 41)}$. In recent years, the H-reflex amplitude has often been recorded after reaching the plateau level at stimulus 
frequencies from 0.2 to $0.5 \mathrm{~Hz}$. It is desirable that the spinal motoneuron pool excitability can be certified in a short period to avoid giving motor stress or examining the effects of therapy. The present study indicated specific frequency disinhibition at a stimulus frequency of approximately $5 \mathrm{~Hz}$ (Fig. 6). This disinhibition may correspond to the repetition of stimulus intervals, which coincides with the overresponse period of the recovery curve due to double stimuli. In other words, it can be accepted as a salient feature of the summation of effects.

As mentioned above, the findings of the $\mathrm{H}$ wave induced by consecutive stimuli at the frequency of $5 \mathrm{~Hz}$ are known to mirror clinical symptoms of neurological disorders. It is germane to mention here that the results of $\mathrm{H}$ wave examination can be used for the assessment of treatment effects of therapies using motor stress or other maneuvers.

With a major goal of examining the spinal motoneuron excitability during exercise therapy or physical therapy, a stimulus frequency of approximately $5 \mathrm{~Hz}$, at which the quality of causative neurological disorder can be identified, was administered in a subsequent study. The measurement was made in a short period in order to minimize external environmental stimulations. In conclusion, we have found that repetitive evoked EMG using the stimulus frequency of approximately 5 $\mathrm{Hz}$ is a great value in examining the change in the H-reflex amplitude and has a good possibility of clinical application.

\section{Acknowledgment}

Grateful acknowledgments are made to Prof. Takashi Yamamoto of Laboratory of Exercise Physiology, Dept. of Health Education, Faculty of Physical Education, Chukyo University, and Associate Prof. Junzo Tsujita, Dept. of Physiology I, Hyogo College of Medicine, for their advice throughout the investigation.

\section{REFERENCES}

1) Tanaka R: Spinal reflex. Brain and Nerve, 43: 10031008, 1991 (in Japanese).

2) Etnyre BR, et al: H-reflex changes during static stretching and two variations of Proprioceptive Neuromuscular Facilitation techniques. Electroenceph. Clin Neurophysiol 63: 174-179, 1986.

3) Yanagisawa K, et al: Effect of shoulder PNF starting positions on H-reflex of soleus muscle. Rigaku ryohogaku 16: 19-22, 1989 (in Japanese).
4) Moore MA, et al: Depression of Hoffmann reflexes following voluntary contraction and implication for Proprioceptive Neuromuscular Facilitation Therapy. Phys Ther 71: 321-333, 1991.

5) Leone JA, et al: Effects of tendon pressure on alpha motoneuron excitability in patients with stroke. Phys Ther 68: 475-480, 1988 .

6) Robichaud JA, et al: Effect of air-splint application on soleus muscle motoneuron reflex excitability in nondisabled subjects and subject with cerebrovascular accidents. Phys Ther 72: 176-185, 1992.

7) Kukulka CG, et al: Effects of intermittent tendon pressure on alpha motoneuron excitability. Phys Ther 66: 1091-1094, 1986.

8) Condon SM, et al: Soleus muscle electromyographic activity and ankle dorsiflexion range of motion during four stretching procedures. Phys Ther 67: 24-30, 1987.

9) Morelli M, et al: Changes in H-reflex amplitude during massage of triceps surae in healthy subjects. JOSPT 12: 55-59, 1990.

10) Morelli $\mathrm{M}$, et al: H-reflex modulation during manual muscle massage of human triceps surae. Arch Phys Rehabil 72: 915-919, 1991.

11) Goldberg J, et al: The effect of two intensities of massage on H-reflex amplitude. Phys Ther 72: 449457, 1992.

12) Uebscheit $\mathrm{N}$, et al: Effect of cooling on the ankle jerk and H-response. Phys Ther 50: 1041-1049, 1970.

13) Uebscheit $N$, et al: Effect of cooling on the ankle jerk and $\mathrm{H}$-response in hemiplegic patients. Phys Ther 51: 983-989, 1971.

14) Hugon M, et al: A discussion of the methodology of triceps surae T- and H-reflexes; in New developments in electromyography and clinical neurophysiology. ed. by Desmedt JE vol.3, Karger, Basel, 1973, pp 773-780.

15) Eccles JC, et al: Effects induced in a monosynaptic reflex path by its activation. J Neurophysiol 14: 353 376,1951 .

16) Lloyd DPC, et al: Reflex depression in rhythmically active monosynaptic reflex pathways. J Gen Physiol 40: 409-426, 1957.

17) Okada Y: Fundamental researches on repetitive evoked EMG. II. Chemical process of neuro-muscular transmission. J Physiol Soc Japan 24: 48-54, 1962 (in Japanese).

18) Enoki M: Experimental studies on the amplitude of monosynaptic reflex responses in repetitive evoked electromyography. Part I. Fluctuaion of the amplitude in monosynaptic reflex responses of gastrocnemius muscle. J Nara Med Ass 16: 644-672, 1965 (in Japanese).

19) Hoffmann P: Untersuchung über die Eigenreflexe (Sehnenreflexe) menschlicher Muskeln. Springer Verleg, Berlin. 1922.

20) Magladery JW, et al: Electrophysiological studies of nerve and reflex activity in normal man.V. Excitation 
and inhibition of two-neurone reflexes by afferent impulses in the same nerve trunk. Bull Johns Hopk Hosp 88: 520-537, 1951.

21) Magladery JW, et al: Electrophysiological studies of nerve and reflex activity in patients with lesions in the nervous system. I. A comparison of spinal motoneurone excitability following afferent nerve volleys in normal persons and patients with upper motor neurone lesions. Bull Johns Hopk Hosp 91: 219-244, 1952.

22) Ookuma Y, et al: Mechanical effect of ankle on the $H$ wave recovery curve of Magladery Method. Jpn J EEG EMG 16: 204, 1988 (in Japanese).

23) Morita $\mathrm{H}$, et al: Change in electric potential of peripheral nerve/muscle by consecutive stimuli. Jpn J EEG EMG 18: 135, 1990 (in Japanese).

24) Honma $S$, et al: Spinal reflex activity in human body. J Physiol Soc Japan 18: 801-802, 1956 (in Japanese).

25) Ono N: Clinical studies on spinal reflex by evoked electromyogram. Handai-Isi 10: 2281-2293, 1958 (in Japanese).

26) Nishii N: Studies on head by repetitive evoked electromyogram. Handai-Isi 10: 25-39, 1958 (in Japanese).

27) Hori Y, et al: Clinical aspects of the repetitive evoked electromyogram. Adv Neurological Sci 3: 413-434, 1959 (in Japanese).

28) Ito Y: Studies on the state of increased tonus of muscle (Rigidity and Spasm) by the Repetitive Evoked Electromyography. Handai-Isi 12: 659-680, 1960 (in Japanese).

29) Masaoka A: Electromyographical studies of m.gastrocnemius and m.soleus on involuntary movements. Handai-Isi 12: 807-827, 1960 (in Japanese).

30) Enoki M: Experimental studies on the amplitude of monosynaptic reflex responses in repetitive evoked electromyography. Part II. Influences of electrical stimulation of cerebral motor cortex on monosynaptic reflex responses. J Nara Med Ass 16: 673-698, 1965 (in Japanese).

31) Ioku M, et al: Monosynaptic reflex and its facilitation and inhibition due to neurological disorders. Brain and Nerve 18: 1075-1083, 1966 (in Japanese).

32) Ioku M, et al: Evoked electromyography of upper limbs in patients with whiplash injury. Nippon rinsyo 27: 2377-2382, 1969 (in Japanese).

33) Ioku M: Electromyographic study of neurological disorder (II). Clin Electroencephalography 13: 604-611, 1971 (in Japanese).

34) Ioku M: Analysis of muscle tonus by evoked electromyogram. Saisin Igaku 28: 235-242, 1973 (in Japanese).

35) Ioku M, et al: H-reflex study upon the patients with spinal cord injuries. Clin Electroencephalography 16: 624-631, 1974 (in Japanese).

36) Kurosaka F, et al: Evoked electromyographical study on developmental changes of muscle tonus in cerebral palsy. Sogo Rihabiriteshon 12: 628-632, 1984 (in Japanese).

37) Kurosaka F, et al: Motor development and muscle tone in infants and children with abnormal tone. Jpn J Rehabil Med 22: 305-310, 1985 (in Japanese).

38) Suzuki M, et al: Electrostimuloanalyzer. Japan Medical Journal 1713: 47-51, 1957 (in Japanese).

39) Tanaka R: The Hoffmann reflex. A neurophysiological technique in humans. J Physiol Soc Japan 48: 719-734, 1986 (in Japanese).

40) Murakosi K: The basic study and clinical application of electromyogram. Advance in Neurological Sci 7: 352-374, 1963 (in Japanese).

41) Fujiwara T, et al: Varying factors for muscle discharge of human spinal reflex. Rigaku ryohogaku 15: 50, 1988 (in Japanese). 